\title{
COMPUTABLE STRONGLY ERGODIC RATES OF CONVERGENCE FOR CONTINUOUS-TIME MARKOV CHAINS
}

\author{
YUANYUAN LIU ${ }^{\otimes 1}$, HANJUN ZHANG $^{2}$ and YIQIANG ZHAO ${ }^{3}$
}

(Received 5 November, 2007; revised 15 April, 2008)

\begin{abstract}
In this paper, we investigate computable lower bounds for the best strongly ergodic rate of convergence of the transient probability distribution to the stationary distribution for stochastically monotone continuous-time Markov chains and reversible continuoustime Markov chains, using a drift function and the expectation of the first hitting time on some state. We apply these results to birth-death processes, branching processes and population processes.
\end{abstract}

2000 Mathematics subject classification: 60J27, 60J80.

Keywords and phrases: strong ergodicity, convergence rate, birth and death process, branching process, population process.

\section{Introduction}

As a type of traditional ergodicity, strong ergodicity has been studied extensively for many years. The earliest example may be dated back to as early as the 1930s [8] for discrete-time Markov chains. Later, the work was extended to continuous-time Markov processes, and criteria involving drift functions and first hitting time moments were obtained; for a detailed description of these results, see, for example, [1,9] and [12]. For strongly ergodic discrete-time Markov chains, the best (largest) strongly ergodic rate of convergence was obtained in [11] by using spectral conditions. There are also other studies on the estimate of the strongly ergodic rate for continuous-time Markov processes, for example, [7] for finite Markov chains by Nash inequality and $[16,17]$ for Markov processes by the coupling method.

\footnotetext{
${ }^{1}$ School of Mathematics, Railway Campus, Central South University, Changsha, Hunan, 410075, PR China; e-mail: liuyy@csu.edu.cn.

${ }^{2}$ School of Mathematics and Computational Science, Xiangtan University, Xiangtan, 411105, PR China; e-mail: hjzhang001@gmail.com.

${ }^{3}$ School of Mathematics and Statistics, Carleton University, Ottawa, Ontario, K1S 5B6, Canada; e-mail: zhao@math.carleton.ca.

(C) Australian Mathematical Society 2008, Serial-fee code 0334-2700/08
} 
Throughout this paper, we denote by $\mathbb{R}\left(\mathbb{R}_{+}\right)$the real (nonnegative) number set, $\mathbb{Z}_{+}$ the nonnegative integer set and $\mathbb{N}$ the positive integer set. Let $\Phi_{t}$ be a right continuous and time-homogeneous Markov process on a Polish space $E$, endowed with the Borel field $\mathcal{B}(E)$. Denote by $P^{t}(x, \cdot)$ the transition function of $\Phi_{t}$. Suppose that the process $\Phi_{t}$ is ergodic, that is, there exists the unique probability distribution $\pi$ such that

$$
\left\|P^{t}(x, \cdot)-\pi\right\| \rightarrow 0, \quad t \rightarrow \infty
$$

where $\|\mu\|$ denotes the usual total variation norm for a signed measure $\mu$, that is, $\|\mu\|=\sup _{|g| \leq 1}|\mu(g)|$.

The process $\Phi_{t}$ is called strongly ergodic if there exists constants $\gamma \geq 0$ and $\lambda \geq 0$ such that

$$
\sup _{x \in E}\left\|P^{t}(x, \cdot)-\pi\right\| \leq \gamma e^{-\lambda t}
$$

for all $t \geq 0$. For an infinite $E$, without loss of generality, we can require $\gamma \geq 2$ since $\sup _{x \in E}\left\|P^{t}(x, \cdot)-\pi\right\|=2$ when $t=0$. Following Mao [17], define

$$
\alpha(\gamma)=\sup \left\{\begin{array}{l|l}
\lambda>0 & \sup _{x \in E}\left\|P^{t}(x, \cdot)-\pi\right\| \leq \gamma e^{-\lambda t}, \forall t \geq 0
\end{array}\right\}
$$

and $\alpha=\alpha(\infty)=\lim _{\gamma \rightarrow \infty} \alpha(\gamma)$ to be the largest (best) strongly ergodic rate of convergence.

REMARK 1.1. It follows from the definition that $\alpha(\gamma)$ is increasing in $\gamma$, and reaches its maximal value at a finite value of $\gamma$.

For any set $A \in \mathcal{B}(X)$, let $\tau_{A}$ be the first hitting time on $A$, that is,

$$
\tau_{A}=\inf \left\{t \geq 0 \mid X_{t} \in A\right\} .
$$

We write $\tau_{x}=\tau_{\{x\}}$. In [17], a lower bound estimate that $\alpha \geq 1 /\left(\sup _{x \in E} E_{x}\left[\tau_{0}\right]\right)$ was obtained for both one-dimensional diffusion processes and birth-death processes by constructing reflection coupling and classical coupling, respectively. Moreover, an upper bound for a general Markov process was also given in [17] as follows.

PROposition 1.2. If $\Phi_{t}$ is strongly ergodic, then for any closed $A \subset E$ with $\pi(A)>0$,

$$
\alpha(\gamma) \leq\left[\frac{2}{\pi(A)} \log \frac{\gamma}{\pi(A)}\right] \frac{1}{\sup _{x \in E} E_{x}\left[\tau_{A}\right]} .
$$

REMARK 1.3. However, we note that the upper bound depends on $\gamma$ and becomes unbounded as $\gamma$ goes to infinity. This will not be very helpful in bounding the best strongly ergodic rate.

In this paper, we extend the work in [17] and previous literature results to two classes of important continuous-time Markov chains: stochastically monotone 
continuous-time Markov chains (for example, [14]) and reversible continuous-time Markov chains (for example, [4, 5]), by investigating lower bounds on $\alpha$ in terms of drift functions and first hitting time moments.

For the remainder of this paper, let $\Phi_{t}$ be a continuous-time Markov chain on a countable state space $E$. Denote by $Q=\left(q_{i j}\right)$ the $q$-matrix (transition rate matrix) and $P^{t}(i, j)$ the transition function. We assume that $Q$ is totally stable, irreducible and regular. We write $q_{i}=-q_{i i}=\sum_{k \neq i} q_{i k}$ for any $i \in E$.

\section{Stochastically monotone Markov chains}

In the section, we refine computable lower bounds for strongly ergodic rates of convergence for stochastically monotone continuous-time Markov chains, using simple drift functions.

We first provide two equivalent drift conditions that will be used frequently later. (DC1) is in general favorable over (DC2) since it does not involve the parameter $\lambda$. Let $k_{0}$ be an arbitrary state in $E$.

(DC1) Drift Condition 1. There exists a bounded nonnegative solution $y$ of the system

$$
\begin{cases}\sum_{j \in E} q_{i j} y_{j} \leq-1, & i \neq k_{0}, \\ y_{i}=0, & i=k_{0} .\end{cases}
$$

(DC2) Drift Condition 2. For some $\lambda>0$, there exists a bounded solution $V \geq 1$ of the system

$$
\begin{cases}\sum_{j \in E} q_{i j} V_{j} \leq-\lambda V_{i}, & i \neq k_{0}, \\ V_{i}=1, & i=k_{0} .\end{cases}
$$

The equivalence is obvious: if (DC1) holds, then (DC2) holds for $V=y+1$ and any $\lambda \leq 1 /\left(\sup _{i \in E} y_{i}+1\right)$; conversely, if (DC2) holds, then (DC1) holds for $y=\lambda(V-1)$. Note that (DC1) is a well-known sufficient and necessary condition for strong ergodicity (see, for example, [1, Proposition 3.3 in Section 6.3]). We also note that if (DC2) holds, then $\lambda<q_{i}$ is satisfied automatically for any $i \neq k_{0}$ because $0<\sum_{j \neq i} q_{i j} V_{j} \leq\left(q_{i}-\lambda\right) V_{i}$.

The following proposition is essentially the same as [19, Theorem 1] (see also [1, Lemma 1.5 in Section 6.1]) with some minor variations.

\section{PROPOSITION 2.1.}

(i) The sequence $\left\{E_{i}\left[\tau_{k_{0}}\right], i \in E\right\}$ is the minimal nonnegative solution of (2.1) and satisfies the system with equality.

(ii) For some $\lambda$ such that $0<\lambda<q_{i}$ for any $i \neq k_{0}$, the sequence $\left\{E_{i}\left[e^{\lambda \tau_{k_{0}}}\right], i \in E\right\}$ is the minimal nonnegative solution of (2.2) and satisfies the system with equality. 
Proof. (i) The first assertion is the same as that in [1, Lemma 1.5 in Section 6.1].

(ii) From [1, Lemma 1.5 in Section 6.1], we see that the sequence

$$
\left\{\frac{E_{i}\left[e^{\lambda \tau_{k_{0}}}\right]-1}{\lambda}, i \in E\right\}
$$

is the minimal nonnegative solution of the following system

$$
\begin{cases}\sum_{j \in E} q_{i j} x_{j} \leq-\lambda x_{i}-1, & i \neq k_{0} \\ x_{i}=0, & i=k_{0}\end{cases}
$$

Let $V_{i}=\lambda x_{i}+1$, then (2.3) becomes (2.2), and (ii) follows.

We now review the definitions of stochastic monotonicity. The chain $\Phi_{t}$ is said to be stochastically monotone if $\sum_{j \geq k} P^{t}(i, j)$ is an increasing function of $i$ for any fixed $k \in \mathbb{Z}_{+}$and $t \in \mathbb{R}_{+}$. It follows from [20, Theorem 3.1] that $\Phi_{t}$ is stochastically monotone if and only if $Q$ is (stochastically) monotone, that is,

$$
\sum_{j \geq k} q_{i j} \leq \sum_{j \geq k} q_{i+1, j}
$$

for all $i, k \in \mathbb{Z}_{+}$such that $k \neq i+1$.

We let $E=\mathbb{Z}_{+}$in this and the following section. We apply a result in [14] to derive the following lemma. In [14], a stochastically ordered continuous-time Markov process was considered and explicit exponential rates of convergence were found using the coupling method. Note that stochastic monotonicity is equivalent to stochastic ordering for continuous-time Markov chains.

LEMMA 2.2. Assume that $Q$ is monotone. If $\sup _{i \in E} E_{i}\left[e^{\lambda \tau_{0}}\right]<\infty$ for some $\lambda>0$, then

$$
\sup _{i \in E}\left\|P^{t}(x, \cdot)-\pi(\cdot)\right\| \leq 4 \sup _{i \in E} E_{i}\left[e^{\beta \tau_{0}}\right] e^{-\beta t}
$$

for any $0<\beta \leq \lambda$ and $t \geq 0$. Furthermore, if $V$ satisfies (DC2) for some $\lambda>0$, then

$$
\sup _{i \in E} E_{i}\left[e^{\lambda \tau_{0}}\right] \leq \sup _{i \in E} V_{i}<\infty .
$$

PROOF. Since $Q$ is monotone, the chain $\Phi_{t}$ is stochastically monotone (equivalently, stochastically ordered). If $\sup _{i \in E} E_{i}\left[e^{\lambda \tau_{0}}\right]<\infty$, then it follows from [14, Theorem 2.1] that

$$
\left\|P^{t}(i, \cdot)-\pi(\cdot)\right\| \leq M_{i} e^{-\lambda t},
$$

where $M_{i} \leq 2\left(E_{i}\left[e^{\lambda \tau_{0}}\right]+E_{\pi}\left[e^{\lambda \tau_{0}}\right]\right)$. With the fact that

$$
E_{\pi}\left[e^{\beta \tau_{0}}\right]=\sum_{j \in E} E_{j}\left[e^{\beta \tau_{0}}\right] \pi(j) \leq \sup _{i \in E} E_{i}\left[e^{\beta \tau_{0}}\right] \leq \sup _{i \in E} E_{i}\left[e^{\lambda \tau_{0}}\right]
$$

for any $\beta \leq \lambda$, we obtain (2.4) immediately. The second assertion follows from Proposition 2.1 directly. 
ASSUMPTION 2.3. There exists a positive integer $N$ satisfying $q_{n 0}=0$ for $n \geq N$.

Many important classes of $q$-matrices satisfy this assumption, for example, the birth-death $q$-matrix and the branching $q$-matrix that is studied in Section 3.

For a monotone matrix, the following lemma reveals the relationship between (DC1) and (DC2) in more detail.

LEMMA 2.4. Assume that $Q$ is monotone. If (DC1) holds for an increasing function $y$, then (DC2) holds for any $\lambda<1 /\left(\sup _{i \in E} y_{i}\right)$ and the function $V$ defined by

$$
V_{0}=1, \quad V_{i}=c y_{i}, \quad i \geq 1,
$$

where

$$
c=\max \left\{\sup _{i \in E} \frac{q_{i 0}}{1-\lambda y_{i}}, \frac{1}{y_{1}}\right\} .
$$

Furthermore, if $Q$ satisfies Assumption 2.3 and $y$ is finally strictly increasing (that is, there exists some $M \in \mathbb{N}$ such that $y_{i+1}>y_{i}$ for $i \geq M$ ), then (DC2) holds also for $\lambda=1 /\left(\sup _{i \in E} y_{i}\right)$.

Proof. Since $Q$ is monotone, it implies that $q_{i 0}$ is decreasing for $i \geq 1$. By Proposition 2.1(i), we see

$$
y_{1} \geq E_{1}\left[\tau_{0}\right] \geq \frac{1}{q_{1}}>0 .
$$

With the assumption that $y$ is increasing, we know that

$$
c \leq \max \left\{\frac{q_{10}}{1-\lambda \sup _{i \in E} y_{i}}, \frac{1}{y_{1}}\right\}<\infty
$$

for any $\lambda<1 /\left(\sup _{i \in E} y_{i}\right)$.

Let $V_{0}=1$ and $V_{i}=c y_{i}, i \geq 1$, then $V_{i} \geq 1$ for all $i \geq 0$. Now, we check that $V$ satisfies (DC2). For $i \geq 1$, since $c \geq\left(q_{i 0}\right) /\left(1-\lambda y_{i}\right), q_{i 0}-c \leq-\lambda c y_{i}=-\lambda V_{i}$. By this inequality, (DC1) implies that

$$
\sum_{j \in E} q_{i j} V_{j}=q_{i 0}+\sum_{j \geq 1} q_{i j} c y_{j} \leq q_{i 0}-c \leq-\lambda V_{i}, \quad i \geq 1 .
$$

Finally, if Assumption 2.3 holds for $Q$ and $y$ is finally strictly increasing, then

$$
c=\max \left\{\max _{1 \leq i \leq N} \frac{q_{i 0}}{1-\lambda y_{i}}, \frac{1}{y_{1}}\right\}<\infty
$$

even for $\lambda=1 /\left(\sup _{i \in E} y_{i}\right)$, and therefore the conclusion holds.

Based on Lemmas 2.2, 2.4 and the drift condition (DC1), we obtain the following result, which will be applied to stochastically monotone Markov chains. 
THEOREM 2.5. Let $Q$ be a monotone q-matrix. If (DC1) holds for an increasing function $y$, then (1.1) holds for any $\lambda<1 /\left(\sup _{i \in E} y_{i}\right)$ and the constant $\gamma=$ $4 \sup _{i \in E} V_{i}$, where $V$ is given by (2.5). Furthermore, if $Q$ satisfies Assumption 2.3 and $y$ is finally strictly increasing, then (1.1) holds also for $\lambda=1 /\left(\sup _{i \in E} y_{i}\right)$.

Corollary 2.6. Assume that $Q$ is monotone. If $\sup _{i \in E} E_{i}\left[\tau_{0}\right]<\infty$, then

$$
\alpha \geq \frac{1}{\sup _{i \in E} E_{i}\left[\tau_{0}\right]} .
$$

Proof. Let $y_{i}=E_{i}\left[\tau_{0}\right], i \geq 0$. Since the chain $\Phi_{t}$ is stochastically monotone, $y_{i}=E_{i}\left[\tau_{0}\right]$ is increasing of $i$ for $i \geq 0$. By Proposition 2.1(i), we know that $y$ satisfies (DC1) with equality. Hence, the assertion follows from Theorem 2.5.

\section{Applications: stochastically monotone Markov chains}

3.1. Birth-death processes Let $\Phi_{t}$ be a birth-death process with $q$-matrix $Q$ given by

$$
q_{i, i+1}=b_{i}, \quad i \in \mathbb{Z}_{+} ; \quad q_{i, i-1}=a_{i}, \quad i \in \mathbb{N} ; \quad q_{i j}=0, \quad|i-j| \geq 2,
$$

where $a_{i}>0$ for $i \in \mathbb{N}$ and $b_{i}>0$ for $i \in \mathbb{Z}_{+}$. It is well-known that $Q$ is regular if and only if $Q$ is conservative and

$$
R_{b}:=\sum_{n=1}^{\infty}\left(\frac{1}{b_{n}}+\frac{a_{n}}{b_{n} b_{n-1}}+\cdots+\frac{a_{n} \cdots a_{2}}{b_{n} \cdots b_{1}}\right)=\infty .
$$

Define the potential coefficients $\mu_{i}, i \geq 0$, by

$$
\mu_{0}=1, \quad \mu_{i}=\frac{b_{0} b_{1} \cdots b_{i-1}}{a_{1} a_{2} \cdots a_{i}}, \quad i \geq 1 .
$$

Assume that $\Phi_{t}$ is ergodic, that is,

$$
\sum_{n=0}^{\infty} \frac{1}{\mu_{n} b_{n}} \sum_{k=0}^{n} \mu_{k}=\infty \quad \text { and } \quad \mu=\sum_{n=0}^{\infty} \mu_{n}<\infty
$$

Then, the invariant probability measure $\pi$ exists satisfying $\pi_{i}=\left(\mu_{i} / \mu\right), i \in \mathbb{Z}_{+}$.

Define

$$
A=\sum_{i=0}^{\infty} \frac{1}{\mu_{i} b_{i}} \sum_{j=i+1}^{\infty} \mu_{j}, \quad \text { and } \quad S=\sum_{n=1}^{\infty}\left(\frac{1}{a_{n+1}}+\sum_{k=1}^{n} \frac{b_{k} \cdots b_{n}}{a_{k} \cdots a_{n+1}}\right) .
$$

It is easy to see that $A=S+\left(1 / a_{1}\right)$. It was proved in [21] that the birth-death process is strongly ergodic if and only if $S<\infty$. This result can be also proved differently, for example, using an analytic method in [22] or using the coupling method in [16]. The strongly ergodic rate for this process was also considered in [17]. 
THEOREM 3.1. Assume that the birth-death process $\Phi_{t}$ is strongly ergodic. Then (1.1) holds for any $\lambda \leq 1 / A$ and $\gamma=4 c A$ with $c=\max \left\{1 / y_{1}, a_{1} /\left(1-\lambda y_{1}\right)\right\}$, where $y_{1}$ is given by (3.2).

PROOF. It is easy to check that $Q$ is monotone, so is $\Phi_{t}$. From [22], we know that the function $y$ can be defined by

$$
y_{0}=0, \quad y_{i}=\sum_{k=0}^{i-1}\left(\frac{1}{a_{k+1}}+\sum_{j=k+1}^{\infty} \frac{b_{k+1} \cdots b_{j}}{a_{k+1} \cdots a_{j+1}}\right), \quad i \geq 1,
$$

satisfying (DC1) with equality. Obviously, $y_{i}$ is strictly increasing, $Q$ satisfies Assumption 2.3 and $\sup _{i \in E} y_{i}=A$. Therefore, from Theorem 2.5 we have the assertion.

REMARK 3.2. Theorem 3.1 is an improvement of the result in [17], which shows that $\alpha$ assumes the value $(1 / A)$ when $\gamma=4 A c$.

3.2. Generalized Markov branching processes In this section, we consider a generalized Markov branching process with $q$-matrix $Q$ given by

$$
q_{i j}= \begin{cases}q_{i j}, & j>i=0, \\ -\sum_{k=1}^{\infty} q_{0 k}, & j=i=0, \\ r_{i} p_{j-i+1}, & j \geq i-1 \geq 0, j \neq i, \\ -r_{i}\left(1-p_{1}\right), & j=i \geq 1, \\ 0, & \text { otherwise }\end{cases}
$$

where $r_{i} \geq 0$. For $s \geq 0$, define $P(s)=\sum_{i=0}^{\infty} p_{i} s^{i}-s$. The ordinary ergodicity and exponential ergodicity of this class of branching process were investigated in [6], and the strong ergodicity was considered in [22]. However, estimating the convergence rate, as presented here, was not considered in [22]. From [6], we know that if $M_{1}:=\sum_{k=1}^{\infty} k p_{k} \leq 1$, then $Q$ is regular, and the unique $Q$-process is denoted by $\Phi_{t}$.

ASSUMPTION 3.3. The sequence $r_{i}$ is increasing and $\sum_{j \geq k} q_{0 j} \leq r_{1} \sum_{j \geq k} p_{j}$ for any integer $k \geq 2$.

By the definition of the monotonicity of $q$-matrix, we know that $Q$ is monotone if $Q$ satisfies Assumption 3.3. Many branching processes satisfy this assumption, for example, $q_{0 j}=p_{j}$ and $r_{i}=i^{\theta}, \theta>0$.

TheOrem 3.4. Assume that $Q$ satisfies Assumption 3.3. If

$$
M_{1}<1 \quad \text { and } \sum_{i=1}^{\infty}\left(1 / r_{i}\right)<\infty
$$


then (1.1) holds for any

$$
\lambda \leq \frac{p_{0}-\Gamma}{\sum_{i=1}^{\infty}\left(1 / r_{i}\right)} \quad \text { and } \quad \gamma=4 c \frac{1}{p_{0}-\Gamma} \sum_{i=1}^{\infty} \frac{1}{r_{i}}
$$

with

$$
\Gamma=\sum_{k=1}^{\infty} k p_{k+1} \quad \text { and } \quad c=\max \left\{\frac{1}{y_{1}}, \frac{r_{1} p_{0}}{1-\lambda y_{1}}\right\},
$$

where $y_{1}$ is given by (3.4).

PROOF. Using the results in [20] under the Assumption 3.3 and $M_{1}<1$, we obtain that the branching process $\Phi_{t}$ is stochastically monotone. Let $\Gamma=\sum_{k=1}^{\infty} k p_{k+1}$, then $\Gamma=M_{1}-1+p_{0}$. Hence, $M_{1}<1$ if and only if $\Gamma<p_{0}$. Construct a function $y$ as follows:

$$
y_{0}=0, \quad y_{n}=\frac{1}{p_{0}-\Gamma} \sum_{i=1}^{n} \frac{1}{r_{i}}, \quad n \geq 1 .
$$

Since $r_{i}$ is increasing, $y_{i}-y_{i-1}$ is decreasing for $i \geq 1$. For $i \geq 1$,

$$
\begin{aligned}
\sum_{j \in E} q_{i j} y_{j}+1 & =\sum_{j \in E} q_{i j}\left(y_{j}-y_{i}\right)+1 \\
& \leq r_{i} p_{0}\left(y_{i-1}-y_{i}\right)+\sum_{k=1}^{\infty} k p_{k+1} r_{i}\left(y_{i+1}-y_{i}\right)+1 \\
& =r_{i} p_{0}\left(y_{i-1}-y_{i}\right)+r_{i} \Gamma\left(y_{i+1}-y_{i}\right)+1 \\
& \leq 0 .
\end{aligned}
$$

Thus we have checked that $y$ satisfies (DC1). Obviously, $Q$ satisfies Assumption 3.3 and $y$ is strictly increasing, so the assertion follows from Theorem 2.5.

REMARK 3.5. In (3.3), let $r_{i}=i^{\theta}, \theta>0$. From [3, Lemma 2.2], we know that

$$
E_{i}\left[\tau_{0}\right]=\frac{1}{\Gamma(\theta)} \int_{0}^{1} \frac{1-x^{i}}{P(x)}(-\ln x)^{\theta-1} d x
$$

for any $i \geq 1$, where $\Gamma(\theta)$ is the gamma function. Then $\Phi_{t}$ is strongly ergodic if and only if

$$
D:=\sup _{i \in E} E_{i}\left[\tau_{0}\right]=\frac{1}{\Gamma(\theta)} \int_{0}^{1} \frac{d x}{P(x)(-\ln x)^{1-\theta}}<\infty .
$$

Suppose that $D<\infty$ and that $\sum_{j \geq k} q_{0 j} \leq r_{1} \sum_{j \geq k} p_{j}$ for any $k \in \mathbb{N}$ with $k \geq 2$. Then it follows from Theorem 2.5 that (1.1) holds for any

$$
\lambda \leq \frac{1}{D} \quad \text { and } \quad \gamma=\max \left\{\frac{r_{1} p_{0}}{1-\lambda y_{1}}, \frac{1}{y_{1}}\right\}
$$


with

$$
y_{1}=\frac{1}{\Gamma(\theta)} \int_{0}^{1} \frac{1-x}{P(x)}(-\ln x)^{\theta-1} d x .
$$

\section{Reversible Markov chains}

The lower bound for the best rate $\alpha$ is investigated for the reversible Markov chains in this section. We do so by virtue of their skeleton chains. This idea is often used to extend the discrete-time results to their continuous-time counterparts. We first review the definition of the reversibility.

Suppose that the continuous-time chain $\Phi_{t}$ is ergodic with invariant probability measure $\left(\pi_{i}\right)$. We say that the Markov chain $\Phi_{t}$ is reversible if $\pi_{i} P^{t}(i, j)=$ $\pi_{j} P^{t}(j, i)$ for all $i, j \in E$ and all $t \geq 0$. By [1, Propositions 1.2 and 1.6 in Section 7.1], we know that the process is reversible if and only if $Q$ is symmetric (that is, $\pi_{i} q_{i j}$ $=\pi_{j} q_{j i}$. .

We call an ergodic discrete-time Markov chain reversible if $\pi_{i} P(i, j)=\pi_{j} P(j, i)$ for all $i, j \in E$, equivalently (see, for example, [2])

$$
\sum_{i \in E} P f(i) g(i) \pi(i)=\sum_{i \in E} f(i) P g(i) \pi(i)
$$

for all $f, g \in L^{2}(\pi)$. The chain $\Phi_{n}$ is called positive (for example, refer to [2]) if $\sum_{i \in E} P f(i) f(i) \pi(i) \geq 0$ for all $f \in L^{2}(\pi)$. In [2], the explicit geometric rates of convergence were obtained for a positive, reversible and geometrically ergodic discrete-time Markov chain on a general state space, which are stated in Proposition 4.3. It was pointed out in [2] that any skeleton chain $\Phi_{n h}$ of the reversible continuous-time chain $\Phi_{t}$ is positive. Hence, if $\Phi_{t}$ is reversible, then any skeleton chain of $\Phi_{t}$ is positive and reversible.

For the skeleton chain $\Phi_{n h}$, define $\tau_{i}(h)=h \inf \left\{n \geq 1 \mid \Phi_{n h}=i\right\}$ to be the first hitting time on $i$ and $P^{h}(i, j)=P\left[\Phi_{h}=j \mid \Phi_{0}=i\right]$ to be the transition probability. In order to adopt the idea of skeleton chains, we need the following lemma.

LEMMA 4.1. If $\sup _{i \in E} E_{i}\left[e^{r \tau_{j}}\right]<\infty$ for some $j \in E$ and $r>0$, then there exists some $h_{0}>0$ such that $\sup _{i \in E} E_{i}\left[e^{r \tau_{j}(h)}\right]<\infty$ for all $h$ with $0<h \leq h_{0}$.

Proof. Suppose that $\Phi_{0}=i$. Let $D_{k}$ be the $k$ th sojourn time in $j$ and $W_{k}$ be the length of the interval between the $k$ th exit from $j$ and the next visit to $j$ for the chain $\Phi_{t}$. Note that the $D_{k}$ are independent, and the $W_{k}$ too. Also, the $D_{k}$ are independent of the $W_{k}$. Moreover, $D_{k}$ are identically exponentially distributed with the parameter $q_{j}>0$.

Define

$$
N=\min \left\{n \geq 1 \mid \text { the skeleton chain } \Phi_{n h} \text { is in state } j \text { during the interval } D_{n}\right\}
$$


Since $\tau_{j}(h) \leq \sum_{k=1}^{N-1}\left(D_{k}+W_{k}\right)+h+\tau_{j}$,

$$
\begin{aligned}
E_{i}\left[e^{r \tau_{j}(h)}\right] & \leq e^{r h} E_{i}\left[e^{r \tau_{j}}\right] \sum_{n=1}^{\infty} E_{i}\left[\exp \left[r \sum_{k=1}^{n-1}\left(D_{k}+W_{k}\right)\right] I_{\{N\}}\right] \\
& \leq e^{r h} E_{i}\left[e^{r \tau_{j}}\right] \sum_{n=1}^{\infty} E_{j}\left[\exp \left[r \sum_{k=1}^{n-1}\left(D_{k}+W_{k}\right)\right] I_{\cap_{k=1}^{n-1}\left\{D_{k} \leq h\right\}}\right] \\
& \leq e^{r h} E_{i}\left[e^{r \tau_{j}}\right] \sum_{n=1}^{\infty} E_{j}\left[\exp \left[r\left((n-1) h+\sum_{k=1}^{n-1} W_{k}\right)\right] I_{\cap^{n-1}\left\{D_{k} \leq h\right\}}\right] \\
& =\sum_{n=1}^{\infty} e^{n r h} E_{i}\left[e^{r \tau_{j}}\right] E_{j}\left[\exp \left[r \sum_{k=1}^{n-1} W_{k}\right]\left(1-e^{-q_{j} h}\right)^{n-1}\right. \\
& =E_{i}\left[e^{r \tau_{j}}\right] \sum_{n=1}^{\infty} e^{n r h}\left(E_{j}\left[e^{r W_{1}}\right]\right)^{n-1}\left(1-e^{-q_{j} h}\right)^{n-1} .
\end{aligned}
$$

Note that

$$
E_{j}\left[e^{r W_{1}}\right]=\sum_{k \neq j} \frac{q_{j k}}{q_{j}} E_{k}\left[e^{r \tau_{j}}\right]<\infty
$$

we have

$$
\lim _{h \rightarrow 0} e^{r h} E_{j}\left[e^{r W_{1}}\right]\left(1-e^{-q_{j} h}\right)=0 .
$$

Thus, we can choose suitable $h_{0}$ such that

$$
\sum_{n=1}^{\infty} e^{n r h}\left(E_{j}\left[e^{W_{1}}\right]\right)^{n-1}\left(1-e^{-q_{j} h}\right)^{n-1}<\infty
$$

for all $h$ with $0<h \leq h_{0}$. Taking the supremum over $i$ on both sides of (4.1), we have $\sup _{i \in E} E_{i}\left[e^{r \tau_{j}}(h)\right]<\infty$ for all $h$ with $0<h \leq h_{0}$.

REMARK 4.2. Note that we can choose small enough $h$ such that $\sup _{i \in E} E_{i}\left[e^{r \tau_{j}}(h)\right]$ sufficiently close to the value of $E_{i}\left[e^{r \tau_{j}}\right]$.

The following proposition is a specific form of Theorem 1.3 in Baxendal [2], which is used in the proof to Lemma 4.4.

PROPOSITION 4.3 (Baxendal [2]). Let $\Phi_{n}$ be an irreducible, reversible and positive Markov chain on a countable state space E. Suppose that its transition probability matrix $P=(P(i, j))$ satisfies the following conditions:

(i) $P\left(k_{0}, k_{0}\right)=\beta>0$;

(ii) there exists a finite function $\tilde{V} \geq 1$, and constants $\lambda<1$ and $K<\infty$ satisfying

$$
\sum_{j \in E} P(i, j) \tilde{V}_{j} \leq \begin{cases}\lambda \tilde{V}_{i}, & i \neq k_{0} \\ K, & i=k_{0}\end{cases}
$$


Then whenever $\lambda<\rho<1$,

$$
\left\|P^{n}(i, \cdot)-\pi\right\|_{\tilde{V}} \leq M \tilde{V}(i) \rho^{n}
$$

for all $i \in E$ and a positive constant $M$ that depends only on $\beta, \lambda, K$ and $\rho$, where $\|\mu\|_{\tilde{V}}=\sup _{|f| \leq \tilde{V}}|\mu(f)|$ is a stronger norm than the usual total variation norm.

LEMMA 4.4. Let $\Phi_{t}$ be a reversible Markov chain. If $\sup _{i \in E} E_{i}\left[e^{r \tau_{k_{0}}}\right]<\infty$ for some $r>0$, then (1.1) holds for any $\lambda<r$ with the constant $\gamma$ well-defined in the proof to the lemma.

PROOF. Since $\sup _{i \in E} E_{i}\left[e^{r \tau_{k_{0}}}\right]<\infty$, it follows from Lemma 4.1 that there exists some $h_{0}>0$ such that $\sup _{i \in E} E_{i}\left[e^{r \tau_{k_{0}}(h)}\right]<\infty$ for any $h$-skeleton chain with $0<h \leq h_{0}$. For any fixed $h$ with $0<h \leq h_{0}$, let $\tilde{V}_{k_{0}}=1$ and $\tilde{V}_{i}=E_{i}\left[e^{r \tau_{k_{0}}(h)}\right]$ for $i \neq k_{0}$, then by a similar argument as that in [15, proof of Theorem 5.1],

$$
\sum_{j \in E} P^{h}(i, j) \tilde{V}_{j}= \begin{cases}e^{-r h} \tilde{V}_{i}, & i \neq k_{0}, \\ e^{-r h} E_{k_{0}}\left[e^{r \tau_{k_{0}}(h)}\right], & i=k_{0}\end{cases}
$$

For the continuous-time Markov chain $\Phi_{t}$,

$$
P^{h}\left(k_{0}, k_{0}\right) \geq e^{-q_{k_{0}} h}>0 .
$$

Since the skeleton chain $\Phi_{n h}$ is reversible and positive, by Proposition 4.3

$$
\left\|P^{n h}(i, \cdot)-\pi\right\|_{\tilde{V}} \leq M \tilde{V}_{i} \rho^{n h}
$$

for any $\rho$ with $e^{-r}<\rho<1$ and $M$ depending only on $r, q_{k_{0}}, \rho, h$ and $E_{k_{0}}\left[e^{r \tau_{k_{0}}(h)}\right]$. Hence, for any $t \in \mathbb{R}_{+}$with $t=n h+s$ for some $n \in Z_{+}$and $s \in[0, h]$,

$$
\begin{aligned}
\left\|P^{t}(i, \cdot)-\pi\right\| & \leq\left\|P^{n h}(i, \cdot)-\pi\right\|\left\|P^{s}\right\| \\
& \leq\left\|P^{n h}(i, \cdot)-\pi\right\|_{\tilde{V}} \\
& \leq M \tilde{V}_{i} \rho^{n h+s} \rho^{-s} \\
& \leq M \tilde{V}_{i} \rho^{-h} \rho^{t} .
\end{aligned}
$$

Note that the value of $h$ can be chosen small enough so that $\rho^{h}$ is very close to 1 . Thus,

$$
\sup _{i \in E}\left\|P^{t}(i, \cdot)-\pi\right\| \leq M \sup _{i \in E} \tilde{V}_{i} \rho^{-h} \rho^{t},
$$

which completes the proof.

Based on the above results, we can refine the lower bound for the reversible Markov chains. 
THEOREM 4.5. Let $\Phi_{t}$ be a reversible Markov chain. If $\sup _{i \in E} E_{i}\left[\tau_{k_{0}}\right]<\infty$ then the best rate $\alpha \geq 1 /\left(\sup _{i \in E} E_{i}\left[\tau_{k_{0}}\right]\right)$. Furthermore, if (DC1) holds for a function $y$, then $\alpha \geq 1 /\left(\sup _{i \in E} y_{i}\right)$.

PROOF. For a recurrent continuous-time Markov chain, by [10, Theorem 9.3.4]

$$
E_{i}\left[\left(\tau_{k_{0}}\right)^{n}\right] \leq n !\left(E_{i}\left[\tau_{k_{0}}\right]\right)^{n}
$$

for any $n \in \mathbb{Z}_{+}$. If $\sup _{i \in E} E_{i}\left[\tau_{k_{0}}\right]<\infty$, then

$E_{i}\left[e^{r \tau \tau_{0}}\right]=\sum_{n=0}^{\infty} \frac{r^{n}\left(E_{i}\left[\left(\tau_{k_{0}}\right)^{n}\right]\right)}{n !} \leq \sum_{n=0}^{\infty}\left(r \sup _{i \in E} E_{i}\left[\tau_{k_{0}}\right]\right)^{n}=\frac{1}{1-r \sup _{i \in E} E_{i}\left[\tau_{k_{0}}\right]}<\infty$

for any $r<1 /\left(\sup _{i \in E} E_{i}\left[\tau_{k_{0}}\right]\right)$. From Lemma 4.4 we know that (1.1) holds for any $\lambda<1 /\left(\sup _{i \in E} E_{i}\left[\tau_{k_{0}}\right]\right)$, which follows $\alpha \geq 1 /\left(\sup _{i \in E} E_{i}\left[\tau_{k_{0}}\right]\right)$.

Furthermore, if (DC1) holds, which implies from Proposition 2.1 that

$$
\sup _{i \in E} E_{i}\left[\tau_{k_{0}}\right] \leq \sup _{i \in E} y_{i}<\infty,
$$

then we have $\alpha \geq 1 /\left(\sup _{i \in E} y_{i}\right)$ from the first part of the assertion.

\section{Examples: reversible Markov chains}

5.1. Generalized birth-death processes Let $\Phi_{t}$ be a generalized birth-death process with $q$-matrix $Q=\left(q_{i j}\right)$ specified by

$$
q_{i j}= \begin{cases}h_{j}, & i=0, \\ d_{j}, & j=0, i \geq 1, \\ b_{i}, & j=i+1, i \geq 1, \\ a_{i}, & j=i-1, i \geq 2, \\ -\left(b_{1}+d_{1}\right), & j=i=1, \\ -\left(a_{i}+b_{i}+d_{i}\right), & j=i \geq 2, \\ 0, & \text { otherwise. }\end{cases}
$$

Note that $Q$ is conservative if and only if $h_{0}=-\sum_{i=1}^{\infty} h_{i}$. It is known that $Q$ is regular if and only if

$$
\begin{aligned}
R & :=\sum_{n=1}^{\infty}\left[\frac{1+d_{n}}{b_{n}}+\frac{a_{n}\left(1+d_{n-1}\right)}{b_{n} b_{n-1}}+\cdots+\frac{a_{n} \cdots a_{2}\left(1+d_{3}\right)}{b_{n} \cdots b_{2}}+\frac{a_{n} \cdots a_{2}\left(1+d_{1}\right)}{b_{n} \cdots b_{2} b_{1}}\right] \\
& =\infty .
\end{aligned}
$$

Denote by $\hat{Q}$ the birth-death $q$-matrix obtained by deleting all elements in the first row and in the first column, and all $d_{i}$ from the principal diagonal elements. The coefficients $\hat{\mu}_{i}$ are defined by

$$
\hat{\mu}_{1}=1, \quad \hat{\mu}_{i}=\frac{b_{1} b_{2} \cdots b_{i-1}}{a_{2} a_{3} \cdots a_{i}}, \quad i \geq 2 .
$$


It is known that $\hat{Q}$ is symmetric if and only if $\hat{\mu}:=\sum_{i=1}^{\infty} \hat{\mu}_{i}<\infty$. For any positive real number $\hat{\mu}_{0}$, let $\mu_{0}=\hat{\mu}_{0}, \mu_{i}=\hat{\mu}_{i}$ for $i \geq 1$ and $\mu=\sum_{i=0}^{\infty} \mu_{i}$. If $\hat{\mu}<\infty$, then $Q$ is symmetric with $\left(\pi_{i}\right)$, where $\pi_{i}=\mu_{i} / \mu$ for $i \geq 0$, as its symmetrizing measure if and only if $h_{i} \pi_{0}=d_{i} \pi_{i}, \quad i \geq 1$.

Following the same notation as that in [22], define

$$
\begin{gathered}
q_{1}^{(0)}=d_{1}, \quad q_{n}^{(k)}=d_{n}, \quad k=0,1, \ldots, n-2, \quad q_{n}^{(n-1)}=d_{n}+a_{n}, \quad n \geq 2, \\
F_{0}^{(0)}=1, \quad F_{n}^{(0)}=\frac{1}{b_{n}} \sum_{k=0}^{n-1} q_{n}^{(k)} F_{k}^{(0)}, \quad n \geq 1, \\
c_{0}=0, \quad c_{n}=\frac{1+\sum_{k=0}^{n-1} q_{n}^{(k)} c_{k}}{q_{n, n+1}}, \quad n \geq 1 .
\end{gathered}
$$

We then have the following bounds for the best rate $\alpha$.

THEOREM 5.1. The $Q$-process $\Phi_{t}$ is strongly ergodic if and only if

$$
D:=\sup _{k \in Z_{+}} \sum_{n=0}^{k}\left(F_{n}^{(0)} c-c_{n}\right)<\infty,
$$

where

$$
c=\sup _{k \in Z_{+}}\left[\frac{\sum_{n=0}^{k} c_{n}}{\sum_{n=0}^{k} F_{n}^{(0)}}\right] .
$$

Furthermore, if $\Phi_{t}$ is reversible, then the best rate $\alpha \geq(1 / D)$.

PROOF. Note that the only difference between $Q$ and a single-birth $q$-matrix (5.1) is in the first row. Therefore, for any state $i \neq 0$, the expectation $E_{i}\left[\tau_{0}\right]$ can be calculated according to the formula for the single-birth process, which is given, based on the [22, proof of Lemma 2.1], by

$$
E_{k}\left[\tau_{0}\right]=\sum_{n=0}^{k}\left(F_{n}^{(0)} c-c_{n}\right)
$$

It follows from Proposition 2.1 and (DC1) that $\Phi_{t}$ is strongly ergodic if and only if $D=$ $\sup _{k \in E} E_{k}\left[\tau_{0}\right]<\infty$. The last assertion follows immediately from Theorem 4.1.

Condition (5.2) looks complicated. In the following, we provide a simpler sufficient condition for strong ergodicity by comparing it with a birth-death process.

THEOREM 5.2. Let $S$ and A be given by (3.1). If $S<\infty$, then $\Phi_{t}$ is strongly ergodic. Furthermore, if $\Phi_{t}$ is reversible, then the best rate $\alpha \geq 1 / A$.

PROOF. If $S<\infty$, then a bounded increasing solution to the equation in (DC1) for $\hat{Q}$ can be constructed as in (3.2). Obviously, for $i \geq 1$

$$
\sum_{j=0}^{\infty} q_{i j} y_{j}=-d_{i} y_{i}+\left[b_{i} y_{i+1}-\left(a_{i}+b_{i}\right) y_{i}+a_{i} y_{i-1}\right] \leq-1 .
$$


Thus, $\left(y_{i}\right)$ is also a solution to the equation in (DC1) for $Q$. Hence, $\Phi_{t}$ is strongly ergodic. The last assertion follows immediately from Theorem 4.5.

5.2. Open Markov population processes Let $B=\mathbb{Z}_{+}$and denote by $B^{m}$ the set of all $m$-dimensional vectors $\boldsymbol{i}=\left(i_{1}, i_{2}, \ldots, i_{m}\right)$ whose components $i_{k} \in B, 1 \leq k \leq m$, are nonnegative integers. Here $i$ is viewed as the population vector of a system consisting $m$ colonies with $i_{k}$ as the population of the $k$ th colony. Given integers $p$ and $q$, with $1 \leq p \neq q \leq m$, define the operators $T_{p}^{+}, T_{p}^{-}$and $T_{p q}$ on $B^{m}$ by

$$
T_{p}^{+}\left(i_{1}, i_{2}, \ldots, i_{m}\right)=\left(i_{1}, i_{2}, \ldots, i_{p}+1, \ldots, i_{m}\right),
$$

and provided $i_{p} \geq 1$,

$$
T_{p}^{-}\left(i_{1}, i_{2}, \ldots, i_{d}\right)=\left(i_{1}, i_{2}, \ldots, i_{p}-1, \ldots, i_{m}\right)
$$

and

$$
T_{p q}\left(i_{1}, i_{2}, \ldots, i_{p}, \ldots, i_{q}, \ldots, i_{d}\right)=\left(i_{1}, i_{2}, \ldots, i_{p}-1, \ldots, i_{q}+1, \ldots, i_{m}\right) .
$$

Definition 5.3. A continuous-time Markov chain $\Phi_{t}$ with the state space $E$ a subset of $B^{m}$ is called a Markov population process if its $q$-matrix $Q$ is given by

$$
q_{\boldsymbol{i} j}= \begin{cases}\alpha_{p}(\boldsymbol{i}) & \text { if } \boldsymbol{j}=T_{p}^{+}(\boldsymbol{i}) \text { for some } p, \\ \beta_{p}(\boldsymbol{i}) & \text { if } \boldsymbol{j}=T_{p}^{-}(\boldsymbol{i}) \text { for some } p, \\ \gamma_{p q}(\boldsymbol{i}) & \text { if } \boldsymbol{j}=T_{p q}(\boldsymbol{i}) \text { for some } p \neq q, \\ -\sum_{\boldsymbol{i} \neq \boldsymbol{j}} q_{i \boldsymbol{j}} & \text { if } \boldsymbol{j}=\boldsymbol{i}(\text { to keep } Q \text { conservative }), \\ 0 & \text { otherwise, }\end{cases}
$$

where the functions $\alpha_{p}(\boldsymbol{i}), \beta_{p}(\boldsymbol{i})$ and $\gamma_{p q}(\boldsymbol{i})$ are nonnegative with the latter two vanishing for $i_{p}=0$. The Markov population process is called open if there exists a vector $\boldsymbol{I}=\left(I_{1}, I_{2}, \ldots, I_{m}\right)$ where some or all of the components may be infinite, such that

$$
E=\left\{\boldsymbol{i} \in B^{m} \mid i_{1} \leq I_{1}, i_{2} \leq I_{2}, \ldots, i_{m} \leq I_{m}\right\} .
$$

For any $n \geq 0$, denote by $E_{n}$ the level subset $\left\{\boldsymbol{i} \in E \mid i_{1}+i_{2}+\cdots+i_{m}\right\}$ of $E$. Here $\left\{E_{n}, n \in Z_{+}\right\}$is a partition of the space $E$ such that $E_{0}=\{\mathbf{0}\}=\{(0,0, \ldots, 0)\}$ and $\sum_{n=0}^{\infty} E_{n}=E$. Define

$$
b_{n}=\max \left\{\sum_{p=1}^{m} \alpha_{p}(\boldsymbol{i}) \mid \boldsymbol{i} \in E_{n}\right\} \quad \text { and } \quad a_{n}=\min \left\{\sum_{p=1}^{d} \beta_{p}(\boldsymbol{i}) \mid \boldsymbol{i} \in E_{n}\right\} .
$$

Consider a birth-death process with birth coefficients $b_{n}$ and death coefficients $a_{n}$. Define $R_{b}, A$ and $S$ as in Section 3.1. The population process was studied by comparing it with the birth-death process (for example, [18]). By [1, Proposition 3.1 in Section 9.3], we know that if $R_{b}=\infty$, then $Q$ is regular and $\Phi_{t}$ is unique. In [13], it was shown that under some conditions on $Q$, the chain $\Phi_{t}$ is reversible. 
THEOREM 5.4. If $S<\infty$, then the $Q$-process $\Phi_{t}$ is strongly ergodic. Moreover, if $\Phi_{t}$ is reversible, then $\alpha \geq 1 / A$.

PROOF. Since $S<+\infty$, the $Q_{b}$-process is strongly ergodic. As $\left\{y_{n}, n \in \mathbb{Z}_{+}\right\}$defined by (3.2) is a bounded nonnegative and increasing solution to the following equation:

$$
b_{n}\left(y_{n+1}-y_{n}\right)=a_{n}\left(y_{n}-y_{n-1}\right)-1, \quad n \geq 1 .
$$

Then for $\boldsymbol{i} \in E_{n}, n \geq 1$,

$$
\sum_{p=1}^{m} \alpha_{p}(\boldsymbol{i})\left(y_{n+1}-y_{n}\right) \leq \sum_{p=1}^{m} \beta_{p}(\boldsymbol{i})\left(y_{n}-y_{n-1}\right)-1,
$$

which, by letting $z_{i}=y_{n}$, leads to

$$
\begin{gathered}
\sum_{p=1}^{m} \alpha_{p}(\boldsymbol{i}) z_{T_{p}^{+}(\boldsymbol{i})}+\sum_{p=1}^{m} \beta_{p}(\boldsymbol{i}) z_{T_{p}^{-}(\boldsymbol{i})}+\sum_{p=1}^{m} \sum_{q=1}^{m} \gamma_{p q}(\boldsymbol{i}) z_{T_{p q}(\boldsymbol{i})}+1 \\
\leq\left(\sum_{p=1}^{m} \alpha_{p}(\boldsymbol{i})+\sum_{p=1}^{m} \beta_{p}(\boldsymbol{i})+\sum_{p=1}^{m} \sum_{q=1}^{m} \gamma_{p q}(\boldsymbol{i})\right) z_{\boldsymbol{i}}
\end{gathered}
$$

or

$$
\sum_{j \in B^{m}} q_{i j} z_{j} \leq-1, \quad \boldsymbol{i} \neq \mathbf{0}
$$

Thus, we have checked that $z$ with $z_{\mathbf{0}}=\mathbf{0}$ is a solution to (DC1). Since

$$
\sup _{i \in E} z_{i}=\sup _{n \in \mathbb{Z}_{+}} y_{n}=A<\infty .
$$

Here $\Phi_{t}$ is strongly ergodic. The last assertion follows from Theorem 4.5.

\section{Acknowledgements}

This work was supported in part by NSERC/MITACS grants of Canada, National Natural Science Foundation of China (Grant No. 10671212), and the Australian Research Council Centre of Excellence for Mathematics and Statistics of Complex Systems.

\section{References}

[1] W. J. Anderson, Continuous-time Markov chains. An applications-oriented approach (SpringerVerlag, New York, 1991).

[2] P. H. Baxendal, "Renewal theory and computable convergence rates for geometrically ergodic Markov chains", Ann. Appl. Probab. 15 (2005) 700-738.

[3] A. Y. Chen, "Ergodicity and stability generalized Markov branching processes with resurrection", J. Appl. Probab. 39 (2002) 786-803. 
[4] M. F. Chen, "Estimation of spectral gap for Markov chains", Acta Math. Sin. New Ser. 12 (1996) 337-360.

[5] M. F. Chen, "Equivelence of exponential ergodicity and $L^{2}$-exponential convergence for Markov chains", Stochastic Process Appl. 87 (2000) 281-297.

[6] R. R. Chen, "An extended class of time-continuous branching processes", J. Appl. Probab. 34 (1997) 14-23.

[7] P. Diaconis and L. Saloff-Coste, "Nash's inequality for finite Markov chains", J. Theor. Probab. 9 (1996) 459-510.

[8] W. Doeblin, "Sur les propriétés asymptotiquea du mouvement régis par certain types de chaine simples", Bull. Math. Soc. Roum. Sci. 39 (1937) 57-115.

[9] D. Down, S. P. Meyn and R. L. Tweedie, "Exponential and uniform ergodicity of Markov processes", Ann. Probab. 23 (1995) 1671-1691.

[10] Z. T. Hou and Q. F. Guo, Homogeneous denumerable Markov processes (Springer, New York, 1988).

[11] D. Isaacson and G. R. Luecke, "Strongly ergodic Markov chains and rates of convergence using spectral conditions", Stochastic Process Appl. 7 (1978) 113-121.

[12] D. Isaacson and R. L. Tweedie, "Criteria for strong ergodicity for Markov chains", J. Appl. Probab. 15 (1978) 87-95.

[13] J. F. C. Kingman, "Markov population processes", J. Appl. Probab. 6 (1969) 1-18.

[14] R. B. Lund, S. P. Meyn and R. L. Tweedie, "Computable exponential convergence rates for stochastically ordered Markov processes", Ann. Appl. Probab. 6 (1996) 218-237.

[15] R. B. Lund and R. L. Tweedie, "Geometric convergence rates for stochastically ordered Markov chains", Math. Oper. Res. 20 (1996) 182-194.

[16] Y. H. Mao, "Strong ergodicity for Markov processes by coupling methods", J. Appl. Probab. 39 (2002) 839-852.

[17] Y. H. Mao, "Convergence rates in strong ergodicity for Markov processes", Stochastic Process Appl. 116 (2006) 1964-1976.

[18] G. E. H. Reuter, "Competition processes", Proc. Fourth Berkeley Symp. Math. Statist. Probab. 2 (1961) 421-430.

[19] R. L. Tweedie, "Criteria for ergodicity, exponential ergodicity and strong ergodicity of Markov processes", J. Appl. Probab. 18 (1981) 122-130.

[20] H. J. Zhang and A. Y. Chen, "Stochastic comparability and dual $q$-functions", J. Math. Anal. Appl. 234 (1999) 482-499.

[21] H. J. Zhang, A. Y. Chen, X. Lin and Z. T. Hou, "Strong ergodicity of monotone transition functions", Statist. Probab. Lett. 55 (2001) 63-69.

[22] Y. H. Zhang, "Strong ergodicity for single-birth processes", J. Appl. Probab. 38 (2001) 270-277. 